\title{
Special Issue on Electronic Systems and Energy Harvesting Methods for Automation, Mechatronics and Automotive
}

\author{
Paolo Visconti *(D), Nicola Ivan Giannoccaro (D) and Roberto de Fazio (D) \\ Department of Innovation Engineering, University of Salento, 73100 Lecce, Italy; \\ ivan.giannoccaro@unisalento.it (N.I.G.); roberto.defazio@unisalento.it (R.d.F.) \\ * Correspondence: paolo.visconti@unisalento.it; Tel.: +39-832-29-7334
}

check for

updates

Citation: Visconti, P.; Giannoccaro, N.I.; de Fazio, R. Special Issue on Electronic Systems and Energy Harvesting Methods for Automation, Mechatronics and Automotive. Energies 2021, 14, 8050. https:// doi.org/10.3390/en14238050

Received: 27 October 2021 Accepted: 25 November 2021 Published: 1 December 2021

Publisher's Note: MDPI stays neutral with regard to jurisdictional claims in published maps and institutional affiliations.

Copyright: (c) 2021 by the authors. Licensee MDPI, Basel, Switzerland. This article is an open access article distributed under the terms and conditions of the Creative Commons Attribution (CC BY) license (https:// creativecommons.org/licenses/by/ $4.0 /)$.

\section{Introduction}

Electronic apparatus have become essential components of civil and industrial systems, including the automotive, home and building automation, Industrial IoT (Internet of Things) and control applications, and playing an essential role in improving security, efficiency, manageability, and rapid feedback [1-3]. Indeed, the increasing demands of electronic systems have led to innovations and findings in electronic networks for automotive and automation plants, replacing efficiently and securely mechanical and hydraulic sections [4]. Also, the researchers have focused their attention on meeting the increasing power demand of vehicles equipment, developing $42-\mathrm{V}$ automotive systems.

Moreover, smart buildings and homes represent a very actual research topic in the scientific community, aimed to improve energy conservation and the liveability of everyday life environments, thanks to IoT solutions [5]. In fact, smart homes and buildings comprise innovative solutions enabling communication between users and the infrastructure, as well as performing advanced monitoring tasks, like surveillance, light and water management, HVAC (heating, ventilation and air conditioning) system management, smart energy monitoring and elderly care. IoT technology employs sensors to detect the environmental temperature for the HVAC system, water and energy consumption, and health monitoring and decision-making systems to assist elderly people and detect fires [6,7]. The scientific community is concentrating their efforts to design innovative infrastructures, management models as well as operating scenarios to make production activities simpler and more efficient [8]. In this field, IoT is one of the key elements triggering this revolution, enabling communications between machines (M2M), thus creating a manufacturing environment human-free. The combination of M2M, IoT and CPS (cyber physical systems) makes the manufacturing systems more robust, reliable and efficient. Besides, cloud computing constitutes a powerful tool, promising to solve several difficult issues with previous productive architectures. For instance, in [9], a novel architecture integrating cloud computing, IoT, and smart devices, was presented. The model uses modern manufacturing technologies, allowing highly configurable, flexible manufacturing processes involving human and robotic participants.

Sensors are crucial devices in several application fields to transduce physical quantities into electrical signals, making them suitable for acquisition and processing systems. Automotive sensors must comply with different requirements, considering the harsh operating conditions (high temperature and humidity, intense vibrations, dust and smoke, etc.). They must be chosen as a trade-off between accuracy, robustness, manufacturability, interchangeability, and low cost [10]. They are placed in different positions of the car, such as in the power train to detect emissions, improve fuel consumption and diagnose engine issues, in the chassis system for improving the vehicle's security(e.g., collision avoidance, ABS-anti-blocking systems, and tire pressure control), and in the body system for comfort and safety applications (e.g., airbag, rollover and side crash protection). Also, systems for 
detecting unattended children abandoned in the car have been discussed in recent years, given the current events [11].

Another topic treated by the proposed special issue regards the application of energy harvesting solutions to different application fields, including automotive, smart building and manufacturing systems. Harvesting sections scavenge energy from available environmental or custom sources, making sensing or actuating devices energetically autonomous or extending their lifetime. In vehicles, a significant fraction $(\approx 77.5 \%)$ of consumed energy is lost in different forms, such as thermal, friction, pump, incomplete combustion, etc., reducing efficiency. Also, vibration reduces the vehicle efficiency, along with causing failure of the mechanical components. Several efforts were addressed to scavenge energy from mechanical vibrations of vehicles, exploiting electromagnetic or piezoelectric transduction mechanisms [12]. Besides, significant energy loss in vehicles is related to braking, dissipating the kinetic energy as heat due to friction. Several regenerative braking systems have been proposed in the scientific literature, using the electromagnetic transduction mechanism [13].

Moreover, energy harvesting systems are applied to supply sensor nodes in industrial environments to monitor production processes, making them energetically autonomous. Different energy sources can be exploited according to the application scenario, like heat, light energy, fluid fluxes, and vibrations [14]. Similarly, smart buildings and homes need a wide range of sensors placed in different structure locations for different applications such as structure monitoring, to monitor human activity, to help in disaster management, e.g., fires and earthquakes [15]. Several energy harvesting sources can supply sensor nodes, such as solar or artificial light, mechanical vibrations, temperature gradients, and radiofrequency signals.

This Special Issue aimed to cover a wide range of disciplines and application fields, collecting innovative studies on advanced sensing and energy harvesting technologies and applications in automotive, automation and mechatronics fields. The introduced innovations could mitigate the impact of human activities on the environment and revolutionize the production process by employing eco-sustainable production models, preventing climate change and natural resources waste.

A total of 5 papers have been published in this special issue; the paper covers a wide range of topics but is deemed relevant to the topics covered by the special issues. The authors are from geographically distributed countries such as Italy, Mexico, Spain, and China. This reflects the great impact of the proposed topic and the effective organization of the guest editorial team of this special issue.

\section{A Short Review of the Contributions in This Issue}

The exhaust gas produced by internal combustion engines represents a potential energy source for mechanical harvesting systems to feed low power electronic sections for remote sensing. Specifically, in [16], the authors characterized the exhaust gas produced by AUDI A4, 2000cc, TDI, diesel engine as a function of temperature, pressure, gas speed, mass flow rate in order to determine the most favourable operating point for energy harvesting. The engine characterization allowed the experimental definition of the operative points, checking all the control unit parameters and the pedal percentage, and the ability to identify torque and power in urban, suburban and freeway use. The experimental results demonstrated that thermal energy is the main component in exhaust energy, as well as kinetic energy, the minority fraction of exhaust energy. Furthermore, they proposed a piezoelectric flexible harvester for scavenging energy from the kinetic energy for the exhaust gas flow; it is constituted by a thin polyimide substrate on which is deposited a $1 \mu \mathrm{m}$ thick layer of piezoelectric AlN, responsible for the electro-mechanical conversion during the vortex-induced vibration. The experimental results demonstrated that the piezoelectric harvester could provide a peak power in the order of $\mu \mathrm{W}$ and the mean power of $n W$. 
As described above, vehicles' vibrations represent a significant energy source to be exploited to reduce fuel consumption. Specifically, it has been reported that the energy potential has values ranging from 46 to $7500 \mathrm{~W}$, as function of vehicle typology, pavement conditions, etc. In [17], the authors analyzed the energy recovery potential of two typologies of Energy Harvesting Shock Absorbers (EHSH), namely those based on ball-screw (BSEHSA) structure and cable transmission (CD-EHSA). The first one is composed of a nut and a screw coupled with a generator, whereas the second one relies on a steel cable and a pair of pulleys for converting the translational into rotational motion. The authors presented the mathematical models of the two harvester typologies, along with two correspondent prototypes. The experimental tests were compared with the simulation results, estimation the amount of energy scavenged by both the structures under different operating conditions. Notably, the absorbers were tested on two vehicles' typologies (Renault Twizy and a Ford Focus), demonstrating that the CD-EHSA system can produce between $80-110 \%$ more power than the BS-EHSA. Both transducers can generate between 500-1000W, considering a rough road (class $C$ road), five passengers in a car travelling at $120 \mathrm{~km} / \mathrm{h}$. The main reason because the CD-EHSA structure produces greater power than BS-EHSA lies in its higher internal inertia. Indeed, the CD-EHSA system produces a greater torque on the generator shaft, thus resulting in a higher average current through its coil.

In addition, several control strategies were proposed for reducing the power consumption variable setting suspension systems. Specifically, in the paper titled "Research on Switching Interconnection Modes and Game Control of Interconnected Air Suspension", the authors introduced a novel control system for interconnected air suspension systems based on the logic of switching interconnection modes and game control for the damper [18]. It includes a switching controller for air suspension interconnection modes and a distribution controller for the damping force; the first determines the optimal IASS mode dynamically, calculating the vehicle dynamic performance index in real-time. Besides, based on set IASS mode, the distribution controller adjusts the damper rigidity using a differential game model of vehicle performance. The simulations demonstrated that the controller reduces the vertical acceleration by $18.32 \%$, as well as the dynamic stroke decreases by more than $10 \%$ under straight conditions; also, the roll angle is reduced by $1.26 \%$ during vehicle steering.

In addition, in the scientific work titled "Limitations and Characterization of Energy Storage Devices for Harvesting Applications", the authors studied the limitations and performances of the main energy storage devices commonly used in energy harvesting applications, namely super-capacitors (SC) and lithium polymer (LiPo) batteries [19]. They analyzed the self-discharge phenomenon in different models of super-capacitors as function of initial voltage and charging duration, modelling the physical phenomenon in terms of self-discharge rate and internal resistance. In particular, they demonstrated that for a longer charging duration (i.e., five hours vs. one hour under charge at $5 \mathrm{~V}$ ), a significant reduction of the self-discharge rate is obtained for all tested SCs was verified, ascribable with a better charge distribution and penetration in the electrodes pores in case of longer charging time durations. By analyzing the obtained self-discharge trends, they provided physical interpretations of the self-discharge mechanism in SC, modelling the voltage drop as the superimposition of two exponential trends featured by different time constants. A short-term contribution was ascribed to the charge redistribution into the electrodes pores, but not related to any charge loss, and a long-term contribution attributed to charge leakages through the SC separator layer and parasitic charges transfer between electrodes and electrolyte. Finally, the self-discharge of two $380 \mathrm{mAh}$ Lipo batteries (model LW 752035 ) was studied, demonstrating a reduction of open-circuit voltage of just $0.59 \%$ and only $1.43 \%$ after $124 \mathrm{~h}$.

Furthermore, energy storage devices are a fundamental component of every energy harvesting system; however, these devices present several side effects that reduce the harvesting efficiency. In the paper titled "An Energy Model Using Sleeping Algorithms for Wireless Sensor Networks under Proactive and Reactive Protocols: A Performance 
Evaluation", the authors presented and analyzed different wireless sensor network models applied to five communication protocols: Ad Hoc On-Demand Distance Vector (AODV), Dynamic Source Routing (DSR), Multi-Parent Hierarchical(MPH), Low Energy Adaptive Clustering Hierarchy (LEACH) and Zigbee Tree Routing (ZTR) [20]. The tests indicated the MPH protocol works well in data processing and transmission, as well a in energy saving. Besides, AODV and DSR are very efficient in terms of backup paths and node-tonode connectivity. Finally, the ZTR protocol is featured by low power consumption and high processing rate but is unreliable in unfavourable network conditions or link failures. Considering power consumption, the MPH shows $4 \%$ lower than ZTR protocol, $15 \%$ than AODV, and $6 \%$ than LEACH.

In addition, the authors tested three sleeping algorithms (Modified Sleeping CrownMSC, Timer Sleeping Algorithm-TSA, and Local Energy Information-LEI), obtaining better energy consumption for MSC respect to TSA $(-6 \%)$ and LEI $(-13 \%)$. Afterwards, the proposed algorithms were compared with two standard wireless technologies(Zigbee and $\mathrm{WiFi}$ ). The tests indicated that Zigbee provided better energy performance than WiFi and lower redundancy in the topology links.

\section{Future Trends in Sensing and Harvesting Systems}

The next generation of sensors in automotive applications will be addressed to improve security, efficiency and comfortable drive experience. Many efforts will be spent to obtain a greater level of vehicle automation, integrating intelligent observation systems, ultrasonic sensors and gesture recognition systems. The industrial sensor market will grow significantly in the future $(+9.8 \%)$, involving new classes of temperature sensors, pressure sensors, force sensors, flow sensors, etc. These sensors will be applied to the next generation of manufacturing robotic machines to collect and process the acquired data, making them increasingly intelligent and autonomous.

Automotive energy harvesting systems are expected to be increasingly present in the next-generation vehicles, especially electric or hybrid ones, for extending energy autonomy and efficiency. Particular attention will be addressed to innovative regenerative braking systems, shock absorbers, photovoltaic layers, etc.

Sensors nodes will be widely employed for monitoring the productive processes and industrial machinery status, allowing remote monitoring and quick fault detection or diagnosis. In this scenario, compact and efficient energy harvesting sections could ensure the energy autonomy to sensor nodes, reducing their maintenance.

Author Contributions: The authors contributed equally to this work. All authors have read and agreed to the published version of the manuscript.

Acknowledgments: The authors are grateful to the MDPI Publisher for the invitation to act as guest editors of this special issue and are indebted to the editorial staff of "Energies" for the kind co-operation, patience and committed engagement.

Conflicts of Interest: The authors declare no conflict of interest.

\section{References}

1. Rahim, M.A.; Rahman, M.A.; Rahman, M.M.; Asyhari, A.T.; Bhuiyan, M.d.Z.A.; Ramasamy, D. Evolution of IoT-Enabled Connectivity and Applications in Automotive Industry: A Review. Veh. Commun. 2021, 27, 1-15. [CrossRef]

2. Elsisi, M.; Mahmoud, K.; Lehtonen, M.; Darwish, M.M.F. Reliable Industry 4.0 Based on Machine Learning and IoT for Analyzing, Monitoring, and Securing Smart Meters. Sensors 2021, 21, 487. [CrossRef] [PubMed]

3. Sittón-Candanedo, I.; Alonso, R.S.; García, Ó.; Gil, A.B.; Rodríguez-González, S. A Review on Edge Computing in Smart Energy by Means of a Systematic Mapping Study. Electronics 2020, 9, 48. [CrossRef]

4. Leen, G.; Heffernan, D. Expanding Automotive Electronic Systems. Computer 2002, 35, 88-93. [CrossRef]

5. Vijayan, D.S.; Rose, A.L.; Arvindan, S.; Revathy, J.; Amuthadevi, C. Automation Systems in Smart Buildings: A Review. J. Ambient Intell. Human Comput. 2020. [CrossRef]

6. O'Grady, T.; Chong, H.-Y.; Morrison, G.M. A Systematic Review and Meta-Analysis of Building Automation Systems. Build. Environ. 2021, 195, 1-13. [CrossRef] 
7. Froufe, M.M.; Chinelli, C.K.; Guedes, A.L.A.; Haddad, A.N.; Hammad, A.W.A.; Soares, C.A.P. Smart Buildings: Systems and Drivers. Buildings 2020, 10, 153. [CrossRef]

8. Oztemel, E.; Gursev, S. Literature Review of Industry 4.0 and Related Technologies. J. Intell. Manuf. 2020, 31, 127-182. [CrossRef]

9. Erasmus, J.; Grefen, P.; Vanderfeesten, I.; Traganos, K. Smart Hybrid Manufacturing Control Using Cloud Computing and the Internet-of-Things. Machines 2018, 6, 62. [CrossRef]

10. Fleming, W.J. New Automotive Sensors-A Review. IEEE Sensors J. 2008, 8, 1900-1921. [CrossRef]

11. Visconti, P.; de Fazio, R.; Costantini, P.; Miccoli, S.; Cafagna, D. Arduino-Based Solution for In-Car- Abandoned Infants' Controlling Remotely Managed by Smartphone Application. J. Commun. Softw. Syst. 2019, 15, 89-100. [CrossRef]

12. Abdelkareem, M.A.A.; Xu, L.; Ali, M.K.A.; Elagouz, A.; Mi, J.; Guo, S.; Liu, Y.; Zuo, L. Vibration Energy Harvesting in Automotive Suspension System: A Detailed Review. Appl. Energy 2018, 229, 672-699. [CrossRef]

13. Mayrink, S.; Oliveira, J.G.; Dias, B.H.; Oliveira, L.W.; Ochoa, J.S.; Rosseti, G.S. Regenerative Braking for Energy Recovering in Diesel-Electric Freight Trains: A Technical and Economic Evaluation. Energies 2020, 13, 963. [CrossRef]

14. Alegret, R.N.; Aragones, R.; Oliver, J.; Ferrer, C. Exploring IIoT and Energy Harvesting Boundaries. In Proceedings of the IECON 2019—45th Annual Conference of the IEEE Industrial Electronics Society, Lisbon, Portugal, 14-17 October 2019; Volume 1, pp. 6732-6736.

15. Kollias, A.; Begert, C.; Nikolaidis, I. Energy Harvesting in Smart Building Sensing: Overview and a Proof-of-Concept Study. In Handbook of Smart Cities: Software Services and Cyber Infrastructure; Maheswaran, M., Badidi, E., Eds.; Springer International Publishing: Cham, Switzerland, 2018; pp. 355-377. ISBN 978-3-319-97271-8.

16. Madaro, F.; Mehdipour, I.; Caricato, A.; Guido, F.; Rizzi, F.; Carlucci, A.P.; De Vittorio, M. Available Energy in Cars' Exhaust System for IoT Remote Exhaust Gas Sensor and Piezoelectric Harvesting. Energies 2020, 13, 4169. [CrossRef]

17. Bowen, L.; Vinolas, J.; Olazagoitia, J.L. Design and Potential Power Recovery of Two Types of Energy Harvesting Shock Absorbers. Energies 2019, 12, 4710. [CrossRef]

18. Sun, L.; Lin, Y.; Geng, G.; Li, Z.; Jiang, H. Research on Switching Interconnection Modes and Game Control of Interconnected Air Suspension. Energies 2019, 12, 3218. [CrossRef]

19. de Fazio, R.; Cafagna, D.; Marcuccio, G.; Visconti, P. Limitations and Characterization of Energy Storage Devices for Harvesting Applications. Energies 2020, 13, 783. [CrossRef]

20. Del-Valle-Soto, C.; Velázquez, R.; Valdivia, L.J.; Giannoccaro, N.I.; Visconti, P. An Energy Model Using Sleeping Algorithms for Wireless Sensor Networks under Proactive and Reactive Protocols: A Performance Evaluation. Energies 2020, 13, 3024. [CrossRef] 